\title{
Influence of Intravenous Immunoglobulin and Methylpred- nisolone on Cytokines secreted by $T$ Lymphocyte in Children Primary Immune Thrombocytopenia ${ }^{1}$
}

\author{
Jingyao Ma ${ }^{1}$, Zhenping Chen ${ }^{2}$, Runhui Wu ${ }^{3 *}$ \\ ${ }^{1}$ Hematology Oncology Center, Beijing Children's Hospital, Capital Medical University \\ ${ }^{2}$ State Key Laboratory of Stem Cell and Reproductive Biology, Institute of Zoology, Chinese Academy of Sciences, Beijing, China \\ ${ }^{3} \mathrm{Head}$ of Comprehensive Care team of haemophilia, Associate Director of Hematology Oncology Center
}

*Corresponding author: Runhui Wu, Beijing Key Laboratory of Pediatric Hematology Oncology, National Key Discipline of Pediatrics, Ministry of Education, Hematology Oncology Center, Beijing Children's Hospital, Capital Medical University, 56 Nanlishi Road, Beijing, 100045, China, Email: runhuiwu@hotmail.com

\begin{abstract}
Objectives: Our study was designed to explore the Influence of Intravenous Immunoglobulin (IVIG) and Methylprednisolone (MP) on cytokines secreted by T lymphocyte in children Primary Immune Thrombocytopenia (ITP).

Materials and Methods: We enrolled children ITP at the onset of their disease from our department between December 2011 and March 2013. We followed them until 12 months of their whole duration, choosing the patients whose ultimate duration less than 3 months and no recurrence. Cytokines measurement by cytometric bead array included IL-2, IL-4, IL-6, IL-10, TNF, IFN and IL-17. We divided patients into 3 groups according to the treatment they received before testing cytokines level, then we compared $\mathrm{T}$ cell cytokines level among these groups.

Results: We enrolled 62 patients with first diagnosed ITP and ultimate duration was less than 3 months without recurrence ( 38 boys and 24 girls, age range 2-178 months, median 33 months). We found IL-2 level decreased in both groups treated with MP for 1 day $(\mathrm{N}=29, \mathrm{P}=0.042)$ and group in which patients treated with IVIG for 1 day $(\mathrm{N}=$ $18, \mathrm{P}=0.048)$ compared with group without any treatment $(\mathrm{N}=15)$.

Conclusion: As for children ITP, although pathways of MP and IVIG in treating thrombocytopenia were different, they both could decrease IL-2 in children ITP ultimately. As a representative cytokine of TH1 cell, IL-2 has been demonstrated to be important in lymphocyte activation and mobilization. So we considered that MP and IVIG can reduce the T cell activation and production of auto-antibodies by decrease IL-2.
\end{abstract}

Received Date: April 3, 2016

Accepted Date: May 23, 2016

Published Date: May 28, 2016

Citation: $\mathrm{Wu}, \mathrm{R}$., et al. Influence of Intravenous Immunoglobulin and Methylprednisolone on Cytokines secreted by T Lymphocyte in Children Primary Immune Thrombocytopenia ${ }^{1}$ (2016) Int $\mathrm{J}$ Hematol Ther 2(2): 1- 4 .

DOI: $10.15436 / 2381-1404.16 .011$

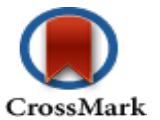

Keywords: ITP; Cytokine; Children; Methylprednisolone; IVIG

\section{Introduction}

Primary Immune Thrombocytopenia (ITP) is characterized by independent thrombocytopenia, which is demonstrated as a disorder mediated by an immune mechanism. Children ITP often have duration of disease within 3 months. Most of them undergo a spontaneous remission, while the remains need treatment to control bleeding. However, there are still $20-30 \%$ patients who finally progress into persistent or chronic stage. Numerous studies prove that auto-antibodies against glycoproteins GPIIb/IIIa, GPIb/IX, etc. can be found in patients diagnosed with ITP. However, not all the ITP patients have positive results in auto-antibodies detection. There might be various mechanisms involved in the onset and progression of ITP. Current studies pay more attention to T lymphocyte abnormalities, including disturbance of TH1/TH2 balance. Studies have shown that patients suffering from ITP have polarized Th1 or Th2 response and cytokine deregulation. The Th1/Th2 balance was well known to regulate the immune system under normal

Copyrights: (C) $2016 \mathrm{Wu}, \mathrm{R}$. This is an Open access article distributed under the terms of Creative Commons Attribution 4.0 International License. 
conditions and to be impaired in autoimmune diseases like ITP. In order to study the mechanism of T lymphocyte abnormalities in children, ITP, we should ensure the homogeneity of the objects. Due to children ITP often have abrupt and severe onset of thrombocytopenia, we prescribe Intravenous Immunoglobulin (IVIG) at the first day of hospitalization to prevent hemorrhage, as well as Methypredisolone(MP), which is often be used when exclusion of malignancy. For this reason, T lymphocyte function evaluation involved detection of cytokines secreted by Th1, Th2 and Th17 etc might be interrupted by treatment we mentioned above. In this study, our objective was to explore the influence of IVIG and MP on cytokines secreted by T lymphocyte in children ITP.

\section{Material and Methods:}

\section{Patients}

We enrolled children, ITP at the onset of their disease without any treatment before hospitalization from Hematology and Oncology Center of Beijing Children Hospital between December 2011 and March 2013 in this prospective clinical study. The diagnosis of ITP was following International consensus report on the investigation and management of primary immune thrombocytopenia. We enrolled ITP patients whose disease duration were less than 3 months and age were between 2 months and 16 years. Patients diagnosed with an infection of Epstein-Barr Virus (EBV), Cytomegalovirus (CMV), hepatitis virus and other pathogens which may potentially induce thrombocytopenia were ruled out. We followed them until 12 months of their whole duration, choosing the patients whose ultimate duration less than 3 months and no recurrence.

We divided them into 3 groups according to the treatment they received before testing cytokines level. Group 1 included patients who received neither MP nor IVIG. Group 2 included those who merely received MP (10mg/ $/ \mathrm{kg} / \mathrm{d})$ for one day and Group 3 included those who merely received IVIG $(0.4 \mathrm{~g} / \mathrm{kg} / \mathrm{d})$ for one day. Informed consent was obtained from participating patients (when old enough) and their parents or guardians. Table 1

Table 1: Groups of different combination of treatment (IVIG and MP)

\begin{tabular}{|l|c|c|c|}
\hline Groups & MP(days) & IVIG (days) & Cases of patients \\
\hline GROUP 1 & 0 & 0 & 15 \\
\hline GROUP 2 & 1 & 0 & 29 \\
\hline GROUP 3 & 0 & 1 & 18 \\
\hline
\end{tabular}

Legend: Group 1 included 15patients who received neither MP nor IVIG. Group 2 included patients who merely received MP for 1 day. Group 3 included patients who merely received IVIG for 1 days.

\section{Plasma Preparation}

We collected blood of all the enrolled patients into evacuated tubes with $1.5 \mathrm{mg} / \mathrm{ml}$ of Ethylenediaminetetraacetic acid (EDTA). All specimens were processed within $2 \mathrm{hrs}$ of collection. Blood samples were centrifuged at $2000 \mathrm{rpm}$ for $10 \mathrm{~min}$ to prepare the plasma. Levels of cytokines secreted by T lymphocyte were analyzed by cytometric bead array (CBA) using FACSCalibur Flow Cytometry from BD (Franklin Lakes, NJ). Cytokines included IL-2, IFN- $\gamma$ and TNF which were mainly secreted by TH1 lymphocyte, IL-4, IL-6 and IL-10 which were mainly secreted by TH2 lymphocyte, IL-17 which were mainly secreted by TH17 lymphocyte. All procedures were carried out at room temperature.

\section{Statistical Analysis}

All statistical analyses were performed using SPSS version 21 (SPSS, Inc., Chicago, IL, USA). Descriptive statistics including the mean and Standard Deviation (S.D) for quantitative variables and number and percentage for qualitative variables were performed. Differences in the cytokine levels among 3 groups were analyzed by One Way ANOVA. A p value of $<0.05$ was considered to be statistically significant.

\section{Results}

\section{Patients}

62 patients of newly diagnosed ITP were enrolled in our study (38 boys and 24 girls, age range 2-178 months, median 33 months). The demographic and clinical characteristics of all enrolled children are summarized in Table 2.

Table 2: Patient's demographic and clinical characteristics.

\begin{tabular}{|l|c|l|c|c|c|c|c|}
\hline Group & N. & Boy/Girl & Age (months) & $\begin{array}{l}\text { Duration } \\
\text { (days) }\end{array}$ & $\begin{array}{l}\text { Min Plat } \\
\text { (109/L) }\end{array}$ & HP positive & $\begin{array}{l}\text { Bleed (No/ } \\
\text { Dry/Wet) }\end{array}$ \\
\hline Total & 62 & $\begin{array}{r}38(61.3 \%) \\
24(38.7 \%)\end{array}$ & $\begin{array}{c}33 \\
(2-178)\end{array}$ & $3(1-42)$ & $7(0-29)$ & $\begin{array}{c}5(11.3 \%) \\
49(79.0 \%) \\
8(13.0 \%)\end{array}$ \\
\hline
\end{tabular}


Levels of cytokines in groups of patients who received different days of IVIG and MP before testing cytokines level: We found IL-2 level decreased in both groups treated with MP for 1 day $(\mathrm{N}=29, \mathrm{P}=0.042)$ and group in which patients treated with IVIG for 1 day $(\mathrm{N}=18, \mathrm{P}=0.048)$ compared with group without any treatment $(\mathrm{N}=15)$. Table 3

Table 3: Cytokines of children ITP patients with treatment of MP for 1 day, IVIG for 1 day and those without treatment before cytokines level been tested.

\begin{tabular}{|l|c|c|c|}
\hline Parameter & No treatment(n=15) & MP for 1 DAY $(\mathbf{n}=\mathbf{2 9})$ & IVIG for 1 DAY $(\mathbf{n}=\mathbf{1 8})$ \\
\hline IL-2 & $4.16 \pm 5.15$ & $1.10 \pm 2.18^{\mathrm{a}^{*}}$ & $1.07 \pm 2.62^{\mathrm{a}^{*}}$ \\
\hline IL-4 & $1.21 \pm 1.28$ & $0.84 \pm 1.20$ & $0.60 \pm 0.95$ \\
\hline IL-6 & $0.59 \pm 0.67$ & $0.64 \pm 1.67$ & $0.64 \pm 0.85$ \\
\hline IL-10 & $4.07 \pm 3.66$ & $4.31 \pm 4.28$ & $3.76 \pm 2.64$ \\
\hline TNF & $2.78 \pm 2.63$ & $3.04 \pm 2.95$ & $2.60 \pm 1.80$ \\
\hline IFN & $0.42 \pm 0.63$ & $0.34 \pm 0.68$ & $1.11 \pm 2.60$ \\
\hline IL-17 & $0.92 \pm 0.93$ & $0.58 \pm 1.66$ & $0.60 \pm 0.72$ \\
\hline
\end{tabular}

a Significance compared to No treatment. $* \mathrm{P}<0.05$ as statistically significant.

\section{Discussion}

ITP is a hemorrhagic disorder of thrombocytopenia without other secondary reasons which could cause decline of platelets. Researchers have found that autoimmune disturbances might be the possible reason for occurrence and development of ITP. Studies showed that patients with ITP demonstrate an increased Th1/Th2 ratio favouring auto-reactive B-cell development ${ }^{[1]}$. As well as the Th17 cells produce cytokines such as IL-17 that may further drive the imbalance between Th1 and Th2 cells, therefore favouring autoimmunity ${ }^{[2]}$. As for children patients, majority of them will remiss within 3 months, while $20-30 \%$ of them develop into a chronic form and have poor life-quality ${ }^{[3]}$. It is eager for us to research in the factors related to the prognosis of children ITP, esp. the immune factors. In order to obtain results with high credibility, we need to exclude the influence of the treatment to the immune factors like cytokines which secreted by T lymphocytes. The standard first-line therapy of ITP was MP and IVIG in our children hospital. So the objective of our study was to explore the influence of IVIG and MP on cytokines secreted by T lymphocyte in children ITP.

MP in symptomatic childhood ITP patients has been suggested as an appropriate first-line therapy ${ }^{[4-7]}$ and it seems wise to use high-dose regimens for as short a period of time as is necessary to achieve a clinically meaningful endpoint ${ }^{[8]}$. MP is a kind of glucocorticoid that prevents the release of substances in the body that cause inflammation. It can mechanically activate the cytosolic glucocorticoid receptor and thus inhibit the pro-inflammatory transcription factor, nuclear factor kappa B (NF-kB). These interactions are important in anti-inflammatory effects of glucocorticoids ${ }^{[9]}$. MP can suppress inflammation by decreasing the release of pro-inflammatory cytokines and vasoactive factors. According to package inserts of SOLU-MEDROL (MP sodium succinate), it has a greater anti-inflammatory potency than prednisolone. After an intravenous infusion of MP, $30 \mathrm{mg} / \mathrm{kg}$ over a $20 \mathrm{minute}$ period, peak plasma concentrations of approximately $20 \mathrm{mcg} / \mathrm{mL}$ were achieved. The mean elimination half-life of MP ranges from 1.8 to 5.2 hours.

IVIG has been reported by Paul Imbach in 1981 that it could increase platelet counts in ITP patients and is considered as their standard therapy ${ }^{[10]}$. IVIG may increase platelet counts in ITP by decreasing FcgR-mediated platelet destruction via competitive-inhibition of Fcg receptors (FcgR), inhibiting complement-mediated platelet elimination, increasing anti-platelet antibody clearance through saturation FcRn, etc ${ }^{[1-12]}$. Rong Deng in 2007 has proved that $43 \pm 5 \%$ of overall effects of IVIG on MWReg30 (anti-platelet antibody) induced thrombocytopenia in mice could be accounted for by the IVIG-mediated increases in MWReg30 clearance $^{[13]}$.

In this study, we found that MP and IVIG both could influence the levels of IL-2. Patients who received MP and IVIG for 1 day had lower levels of IL-2 than those who didn't receive MP. The results proved that although pathways of MP and IVIG in treating thrombocytopenia were different, they both could decrease IL-2 in children ITP ultimately. Several studies have found evidence supporting a Th1 polarization of the immune response in ITP $^{[14-16]}$. Several studies have shown the presence of activated platelet-specific autoreactive $\mathrm{T}$ cells that recognize and respond to autologous platelet antigens and drive the generation of platelet reactive autoantibodies by B cells in the peripheral blood of ITP patients ${ }^{[17-19]}$. As a representative cytokine of TH1 cells, IL-2 has been demonstrated to be important in lymphocyte activation and mobilization ${ }^{[20]}$. So we considered that although pathways of MP and IVIG in treating thrombocytopenia were different, they both could decrease IL-2 in children ITP ultimately. In other words, they both could suppress the activation of T cell and thus re-adjust the TH1/TH2 imbalance, inhibit the destruction lead by $\mathrm{T}$ cell and the generation of antibodies by activated B cell.

In conclusion of our study, as for children, ITP, MP and IVIG for at least 1 day would change patterns of cytokines, esp. level of IL-2 decreased after using MP and IVIG, but the mechanism still need to be prove. Furthermore, we should enlarge our sample size, enroll healthy children as the control group and try our best to acquire datum of the same patients before and after the treatment. 


\section{Reference}

1. Panitsas, F.P., Theodoropoulou, M., Kouraklis, A., et al. Adult chronic idiopathic thrombocytopenic purpura (ITP) is the manifestation of a type1 polarized immune response. (2004) Blood 103(7): 2645-2647.

2. McKenzie, C.G., Guo, L., Freedman, J., et al. Cellular immune dysfunction in immune thrombocytopenia (ITP). (2013) Br J Haematol 163(1): $10-23$.

3. Imbach, P1., Kühne, T., Müller, D., et al. Childhood ITP: 12 months follow-up data from the prospective registry I of the Intercontinental Childhood ITP Study Group (ICIS). (2006) Pediatr Blood Cancer 46(3): 351-356.

4. Ozsoylu, S., Irken, G., Karabent, A. High-dose intravenous methylprednisolone for acute childhood idiopathic thrombocytopenic purpura. (1989) Eur J Haematol 42(5): 431-435.

5. Ozsoylu, S., Sayli, T.R., Ozturk, G. Oral megadose methylprednisolone versus intravenous immunoglobulin for acute childhood idiopathic thrombocytopenic purpura. (1993) Pediatr Hematol Oncol 10(4): 317-321.

6. Barrios, N.J., Humpert, J.R., McNeil, J. Treatment of acute idiopathic thrombocytopenic purpura with higher-dose methylprednisolone and immunoglobulin. (1993) Acta Haematol 89(1): 6-9.

7. George, J.N., Woolf, S.H., Raskob, G.E., et al. Idiopathic thrombocytopenic purpura: a practice guideline developed by explicit methods for the American Society of Hematology. (1996) Blood 88(1): 3-40.

8. Blanchette, V., Bolton-Maggs, P. Childhood immune thrombocytopenic purpura: diagnosis and management. (2010) Hematol Oncol Clin North Am 24(1): 249-273.

9. Garside, H., Stevens, A., Farrow, S., et al. Glucocorticoid ligands specify different interactions with NF-kappaB by allosteric effects on the glucocorticoid receptor DNA binding domain. (2004) J Biol Chem 279(48): 50050-50059.

10. Imbach, P., Barandun, S., d'Apuzzo, V., et al. High-dose intravenous gammaglobulin for idiopathic thrombocytopenic purpura in childhood. (1981) Lancet 1(8232): 1228-1231.

11. Hansen, R.J., Balthasar, J.P. Mechanisms of IVIG action in immune thrombocytopenic purpura. (2004) Clin Lab 50(3-4): 133-140.

12. Jin, F., Balthasar, J.P. Mechanisms of intravenous immunoglobulin action in immune thrombocytopenic purpura. (2005) Hum Immunol 66(4): 403-410.

13. Deng, R., Balthasar, J.P. Pharmacokinetic/pharmacodynamic modeling of IVIG effects in a murine model of immune thrombocytopenia. (2007) J Pharm Sci 96(6): 1625-1637.

14. Zhu, J., Yamane, H., Paul, W.E., et al. Differences in serum cytokine levels in acute and chronic autoimmune thrombocytopenic purpura: relationship to platelet phenotype and antiplatelet T-cell reactivity. (1996) Blood 87: 4245-4254.

15. Andersson, J. Cytokines in idiopathic thrombocytopenic purpura (ITP). (1998) Acta Paediatr Suppl 424: 61-64.

16. Yoshimura, C., Nomura, S., Nagahama, M., et al. Plasma-soluble Fas (APO-1, CD95) and soluble Fas ligand in immune thrombocytopenic purpura. (2000) Eur J Haematol 64: 219-224.

17. Semple, J.W., Lazarus, A.H., Freedman, J. The cellular immunology associated with autoimmune thrombocytopenic purpura: an update. (1998) Transfus Sci 19: 245-251.

18. Kuwana, M., Kaburaki, J., Ikeda, Y. Autoreactive T cells to platelet GPIIb-IIIa in immune thrombocytopenic purpura: role in production of anti-platelet autoantibody. (1998) J Clin Invest 102: 1393-1402.

19. Kuwana, M., Kaburaki, J., Kitasato, H., et al. Immunodominant epitopes on glycoprotein IIb-IIIa recognized by autoreactive T cells in patients with immune thrombocytopenic purpura. (2001) Blood 98: 130-139.

20. Cantrell, D.A., Smith, G., Nelson, D.L. The interleukin-2 T-cell system: A new cell growth model. (1984) Science 224: $1312-1316$.

Ommega Online Publisher

International Journal of Hematology \& Therapy

Short Title : Int J Hematol Ther
ISSN: 2381-1404

E-mail : hematology.therapy@ommegaonline.org website: www.ommegaonline.org 